\title{
A comparison of sedation with midazolam-ketamine versus propofol-fentanyl during Endoscopic retrograde cholangiopancreatography in adults
}

\author{
By \\ Dr.Ghassan Dakhel Ridha Alhajemi* ${ }^{* 1}$; Prof. Dr.Ayad Abbas \\ Salman $^{* 2}$
}

\author{
${ }^{* 1}$ M.B.Ch.B., Dhi Qar Al-Rifa'I hospital; ${ }^{* 2}$ M.B.Ch. B / DA/F. I.C.M.S \\ /CAB\&IC/FIPP,Acting chairman of the Scientific Counsel of Iraqi Board \\ Of Anesthesia and Intensive Care \\ Baghdad-Iraq
}

\begin{abstract}
:
Background: Patients undergoing endoscopic retrograde cholangiopancreatography (ERCP) require adequate sedation or general anesthesia. This prospective clinical study investigated the safety and efficacy of midazolam -Ketamine versus propofol-fentanyl during ERCP procedures. patients and Methods: The study included 80 patients who underwent ERCP following anesthesiologist-administered sedation with fentanyl-propofol and other group midazolam-Ketamine. Procedural data, oxygen saturation, blood pressure, heart rate, recovery time, discharge times the effectiveness of sedation during the procedure was assess according to Modified Observer's Assessment of Alertness/Sedation (MOAA/S) scores. Results: Sedationrelated complications occurred in 13 of 40 patients in group Ketamine midazolam and 5 of 40 patients in group fentanyl-propofol included nausea / vomiting, agitation and headache. Most events were minor and did not necessitate discontinuation of the procedure. Successful cannulation was performed in all patients. the time of recovery was significantly lower in group MK with $5.15 \pm 1.3$ min compared to $6.15 \pm 2$ in group FP, and discharge was significantly. lower in group FP with $20.35 \pm 0.7 \mathrm{~min}$, compared to $24.15 \pm 3.4 \mathrm{~min}$ in group MK. this occurred due to the number of patients who had side effects in midazolam-Ketamine group was more than the other group. Conclusion: We concluded that FP, more suitable for sedation and less complications rather than MK. Although no significant complications were seen.
\end{abstract}

Keywords: sedation, fentanyl -Propofol ,Midazolam-Ketamine 


\section{Introduction:}

Endoscopic retrograde cholangiopancreatography (ERCP) is a technique that combines the use of endoscopy and fluoroscopy to diagnose and treat certain problems of the biliary or pancreatic ductal systems. Through the endoscope, the physician can see the inside of the stomach and duodenum, and inject a contrast medium into the ducts in the biliary tree and pancreas so they can be seen on radiographs ${ }^{(1)}$ The principles governing the optimal use of sedation/anesthesia for ERCP are no different from those for other gastrointestinal endoscopic procedures, but they tend to be more complex and often prolonged, so that optimal conditions are essential to maximize the chances of a successful outcome, and minimize the risks of adverse events The routine use of sedation during endoscopic procedures is increasing worldwide.

In a survey conducted in the United States in 2006, > 98\% of endoscopies and colonoscopies were performed under sedation. A similar trend has been observed in Switzerland, Germany, and Australia. The combination of a benzodiazepine and opioid is reportedly used at approximately $75 \%$ of all healthcare facilities in the United States and considered.The combination of choice by most endoscopists worldwide. As a result of its anxiolytic and sedative properties, its ability to provide anterograde amnesia, and its short half-life, midazolam is the most widely used benzodiazepine. Fentanyl is the most widely used opioid, although meperidine is still frequently used ${ }^{(2)}$. The anesthesia or sedation work area is located at the head of the bed and allows direct access to the patient's airway. There is significant institutional variability to sedation and anesthesia approaches for ERCP. The approaches range from general anesthesia with airway intubation to nurse administered conscious sedation $^{(3)}$.

During sedation and analgesia, there is a continuum of states, ranging from mild sedation to general anesthesia. In the middle of this continuum is conscious sedation, which is the target level of sedation for patients undergoing upper or lower gastro- intestinal endoscopy ${ }^{(2)}$,Most patients undergoing ERCP will require deep sedation or general anesthesia. In this state, patients may respond only to painful stimuli. Additionally, the patients' protective airway reflexes and spontaneous ventilation may become compromised .In a minority of cases, moderate sedation can be employed ${ }^{(4)}$.

Different sedation agents including their potential interactions with other medications and other adverse reactions should be an important prerequisite to procedural sedation. This should include opioids such as meperidine and fentanyl, benzodiazepines such as midazolam and 
diazepam, propofol, pharyngeal anesthetic agents, and potential adjunctive agents such as ketamine, diphenhydramine, promethazine, and droperidol. Additionally, the use of reversal agents such as flumazenil and naloxone should be mastered ${ }^{(4)}$, current study use a combination of midazolam and ketamine versus fentanyl and propofol for sedation of patients undergoing ERCP. Fentanyl is one of the most common parenteral opioid analgesics administered in balanced anesthesia because it allows smooth emergence from anesthesia without coughing and bucking, and provides residual postoperative analgesia ${ }^{(5 \& 6)}$.

Propofol is the most frequently used intravenous (IV) an aesthetic agent It is suitable for the induction and maintenance of an aesthesia. the use of propofol for general anesthesia and sedation has been purported to reduce postoperative emesis and requirements for antiemetic (7\&8). Ketamine is a non barbiturate an aesthetic drug with analgesic effects, which is fast-acting and used for premedication, sedation, induction or maintenance of anesthesia and postoperative analgesia ${ }^{(9)}$. There has been increased interest in the routine use of ketamine in small doses for preventive analgesia and for the treatment or prevention of opiate tolerance a hyperalgesia. $[9,10]$ Benzodiazepines are among the most frequently prescribed drugs. Midazolam is often used for sedation as a premedication for balanced an aesthesia; however, there is often a disparity in the level of sedation compared with the presence of amnesia (10)

\section{Patient and method:}

This was a, clinical study, prospective, randomized clinical trial carried out between (September of 2017) and (September 2018) at the Gastrointestinal Endoscopy ward of the Department of Gastroenterology at the Baghdad teach hospital. The clinical trial was recorded after patient consent was written and approval of scientific counsel of anesthesia and intensive care. We recruited 87 patients from these scheduled to undergo upper gastrointestinal endoscopy at the Gastrointestinal intestinal Endoscopy Unit. The inclusion criteria were: age between 18-65 year, and physical state classifies as ASAI, II.

The exclusion criteria were follows (a history of allergy to the medications to be administered; a history of allergy to soy or eggs; pregnancy; a psychotic disorder, being under treatment with psychoactive medications, being an unlawful drug user or a heavy consumer of alcohol, of the 87 patients recruited, 7 were excluded. The final sample comprised 80 patients. Patients were (one of them inserted 
the endoscope through a trachea, the rest failure to complete sedation with $\mathrm{MK}$ ) randomized to two groups of 40: propofol-fentanyl (FP)and midazolam -ketamine (MK). The. procedures were applied in the endoscopy unit by endoscopist and an endoscopy nurse. Patients followed-up was performs at the recovery room after the procedures. All procedures were performed in left lateral position. After 6-h fasting, check the vital signs, $\mathrm{SPO}_{2}$, then the patients were received midazolam plus ketamine combination in group A or fentanyl plus propofol combination in group B for sedation. The effectiveness of sedation during the procedure was assesst according to Modified Observer's Assessment of Alertness/Sedation (MOAA/S) scores. This scale ranges from 1 to 5 and reflects patient responsiveness alone TABLE MOAA/S (1).

No studies to date have evaluated the impact of frequent measurements (e.g., each 2 moment) of the MOAA/S score on sedation relate outcomes. Since frequent assessments of patient alertness are already mandatory in ERCP, the advantage of MOAA/S is probably limited as a research tool for its ability to allow rapid assessment of sedation depth but not as an instrument for titrating sedation per se. Thought, the MOAA/S is a reminder of the significance of frequent estimations of sedation depth to allow the assessment of imminent sedation-concerting. adverse effects before the onset of a severe adverse effects ${ }^{(4) \text {. }}$

The patients in each group were administered a spray of xylocaine $10 \%$ as a topical pharyngeal anesthesia before sedation. An IV midazolam bolus dose $0,05 \mathrm{mg} / \mathrm{kg}$ [(maximum $3 \mathrm{mg}$ ] was administered to group MK, two minutes later; ketamine bolus dose $0,5-1 \mathrm{mg} / \mathrm{kg}$ was administered intravenously. Patient responses to touch and phonic stimuli were assessed $2 \mathrm{~min}$ after the administration of ketamine. Ketamine $0,5 \mathrm{mg} / \mathrm{kg}$ [max. of $2 \mathrm{mg} / \mathrm{kg}$ ] was added at two minutes intervals if suitable sedation was not done initially. The endoscopy process was started by the endoscopist if the patient as adequate sedated. If the patients were excited after the beginning of the procedure, a single dose of ketamine $0.5 \mathrm{mg} / \mathrm{kg}$ was administered. Maintenance [ 10-45] mic $/ \mathrm{kg} /$ minute, rate adjusts according to level of hypnosis. Other group in addition of topical anesthesia by $10 \%$ xylocaine, Intravenous fentanyl bolus dose 1 microgram $/ \mathrm{kg}$ was administered to group FP, after 2 min; propofol bolus dose $1 \mathrm{mg} / \mathrm{kg}$ was administered IV. Patient responses to touch and phonic stimuli were assessed two minutes 11 after the 
administration of propofol. $0.5 \mathrm{mg} / \mathrm{kg}$ of Propofol was added at two minutes periods if accepted sedation was not approved. The process was beginning by the endoscopist if there was acceptable level of sedation.

If the patients were excited after the procedure beginning, a single dose of propofol $0.5 \mathrm{mg} / \mathrm{kg}$ was administered. Maintenance of sedation by propofol intravenous infusion, $25-100$ micrograms $/ \mathrm{kg} /$ minute dose and rate of administration adjusted according to desired level of sedation.

All patient was monitored for peripheral oxygen saturation $\left(\mathrm{SPO}_{2}\right)$, heart rate (HR) throughout the procedure, blood pressure (every $10 \mathrm{~min}$ and MOAA/S every 2 min. Oxygen supplying by a nasal apparatus was administered to the patients through the process. Cardiac episode, temporary cassation of breath and laryngospasm were monitored as important complications, whereas decrease oxygen saturation $(<90 \%$ during $60 \mathrm{~s}$ ), increase pulse, decrease rate, increase in oral saliva (copious oral secretions requiring suctioning), rasping and nausea and vomiting were assessed as minor complications.

All events were documented. The starting and end of the procedure were recorded after providing sedation. The ending of the process without any major complications denoted the successful sedations. The discharge of the patients from theatre after assessment with Aldrete score. The Aldrete score was set by scoring from 0 to 10 according to the patient's activity, oxygen saturation, consciousness, respiration and circulation ,Patients with an Aldrete score of 9 or greater were discharged from the ERCP unit. The duration between the completion of the procedure and departure from the endoscopy unit (discharge time). During the recovery time complications such as headache, agitation, nausea and vomiting were listed.

\section{Results:}

The total number of patients were 80 , distributed into two equal groups, with no statistically significant difference between age, weight, times of procedure, while the time of recovery was significantly lower in group MK with $5.15 \pm 1.3 \mathrm{~min}$, compared to $6.15 \pm 2$ in group FP, and discharge was significantly lower in group FP with $20.35 \pm 0.7 \mathrm{~min}$, 
compared to $24.15 \pm 3.4$ min in group MK. The gender distribution was not biased between groups, as teen in Table (1).

Table (1): Distribution of age and gender according to the study groups

\begin{tabular}{|c|c|c|c|}
\hline \multirow[t]{2}{*}{ Variables } & Group MK & Group FP & \multirow[t]{2}{*}{ P-value } \\
\hline & Mean \pm SD & Mean \pm SD & \\
\hline Age & $47.15 \pm 13.9$ & $51.46 \pm 13.1$ & $0.160 *$ \\
\hline Weight & $75.93 \pm 8.3$ & $74.18 \pm 7.8$ & $0.333^{*}$ \\
\hline Period of procedure & $24.13 \pm 7.4$ & $26.15 \pm 9.7$ & $0.299^{*}$ \\
\hline Period of recovery & $5.15 \pm 1.3$ & $6.15 \pm 2$ & $0.010 *$ \\
\hline Period of discharge & $24.15 \pm 3.4$ & $20.35 \pm 0.7$ & $<0.001 *$ \\
\hline Gender & No $(\%)$ & No $(\%)$ & \multirow[t]{3}{*}{$0.639 * *$} \\
\hline Male & $15(37.5)$ & $13(32.5)$ & \\
\hline Female & $25(62.5)$ & $27(67.5)$ & \\
\hline Total No & 40 & 40 & - \\
\hline
\end{tabular}

There was no statistically significant association between side effects and study groups, as group MK showed slightly more side effects compared to group FP, as shown in Table (2)

Table (2): Distribution of side effects according to study groups

\begin{tabular}{|l|c|c|c|}
\hline Side effects & No. (\%) & No. (\%) & P value \\
\cline { 1 - 2 } & & & \multirow{2}{*}{0,032} \\
\cline { 1 - 3 } No & $27(67.5)$ & $35(87.5)$ & 1,0 \\
\hline Hypoxia & $1(2.5)$ & $0(0)$ & 0,493 \\
\hline Cough & $2(5)$ & $0(0)$ & 0,240 \\
\hline Nausea/vomiting & $3(7.5)$ & $0(0)$ & 0,709 \\
\hline Agitation & $5(12.5)$ & $3(7.5)$ & 1,0 \\
\hline Headache & $2(5)$ & $2(5)$ & \\
\hline Total No & 40 & 40 & \\
\hline
\end{tabular}


The systolic blood pressure did not show statistically significant differences throughout the anesthesia timeline, except at 10 minutes, where group FP showed lower mean systolic BP, also it can be observed that the systolic BP readings were lower throughout the anesthesia timeline, as shown in figure (1).

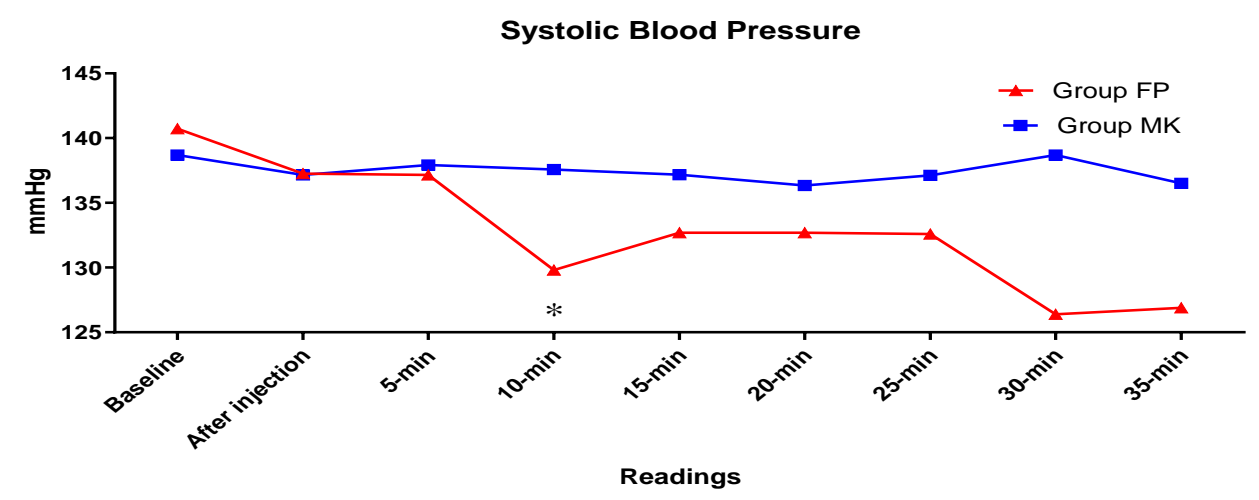

Figure (1): distribution of systolic blood pressure at different intraoperative time intervals according to study groups (the asterisk indicates statistically significant difference, $p$-value $<\mathbf{0 . 0 5}$ )

The diastolic BP started to be significantly lower in group FP compared to group MK at 5-min, then at 15-25 minutes, as shown in Figure (2)

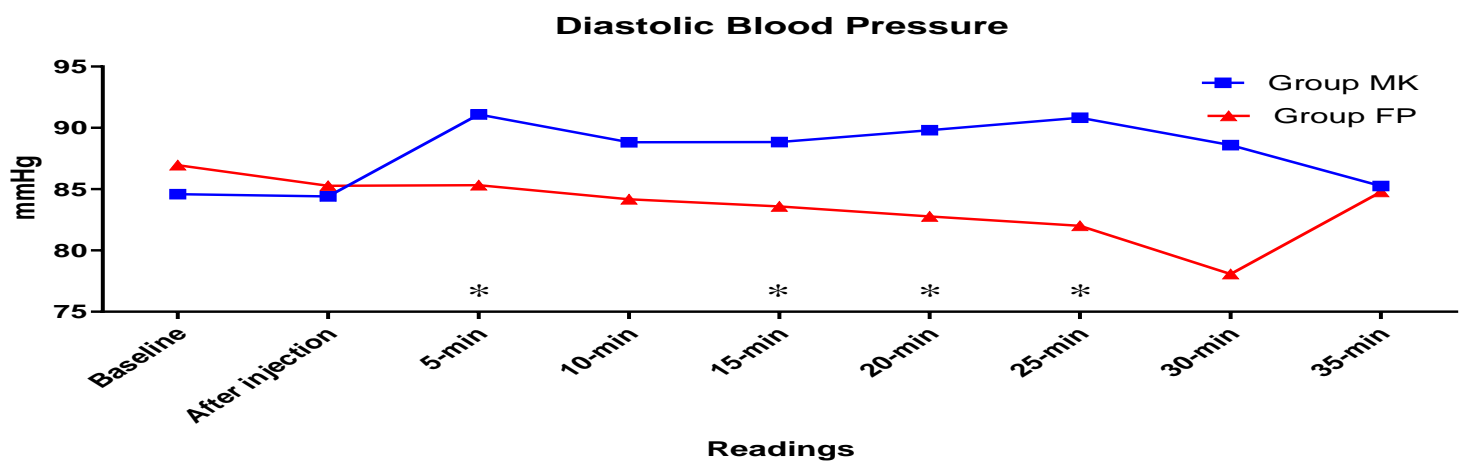

Figure (2): distribution of diastolic blood pressure at different intraoperative time intervals according to study groups (the asterisk indicates statistically significant difference, $p$-value $<\mathbf{0 . 0 5}$ ). 
The MAP showed generally lower values throughout the anesthesia, being significant at 10 and 15 minutes, and comparable stability between the groups, as shown in Figure (3).

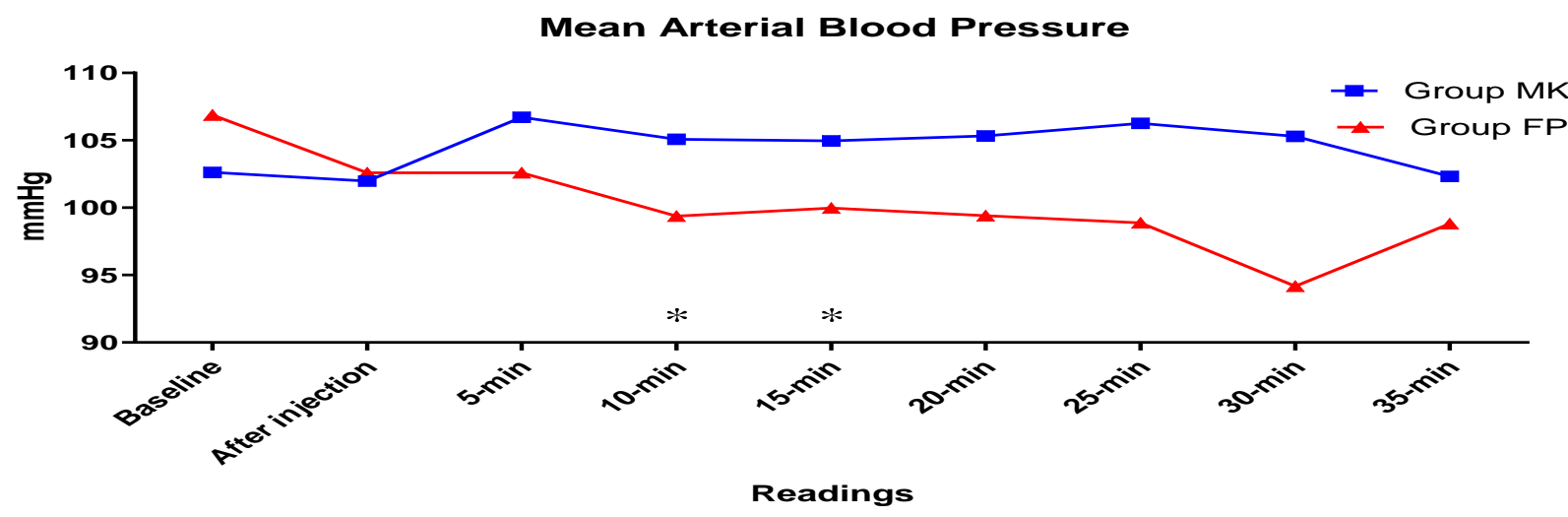

Figure (3): distribution of mean arterial blood pressure at different intraoperative time intervals according to study groups (the asterisk indicates statistically significant difference, $p$-value $<0.05$ ).

The pulse rate was higher in group MK from the start of operation until 20-25 minutes, with significant differences only after injection of MK, or FP and 5-min, as shown in Figure (4).

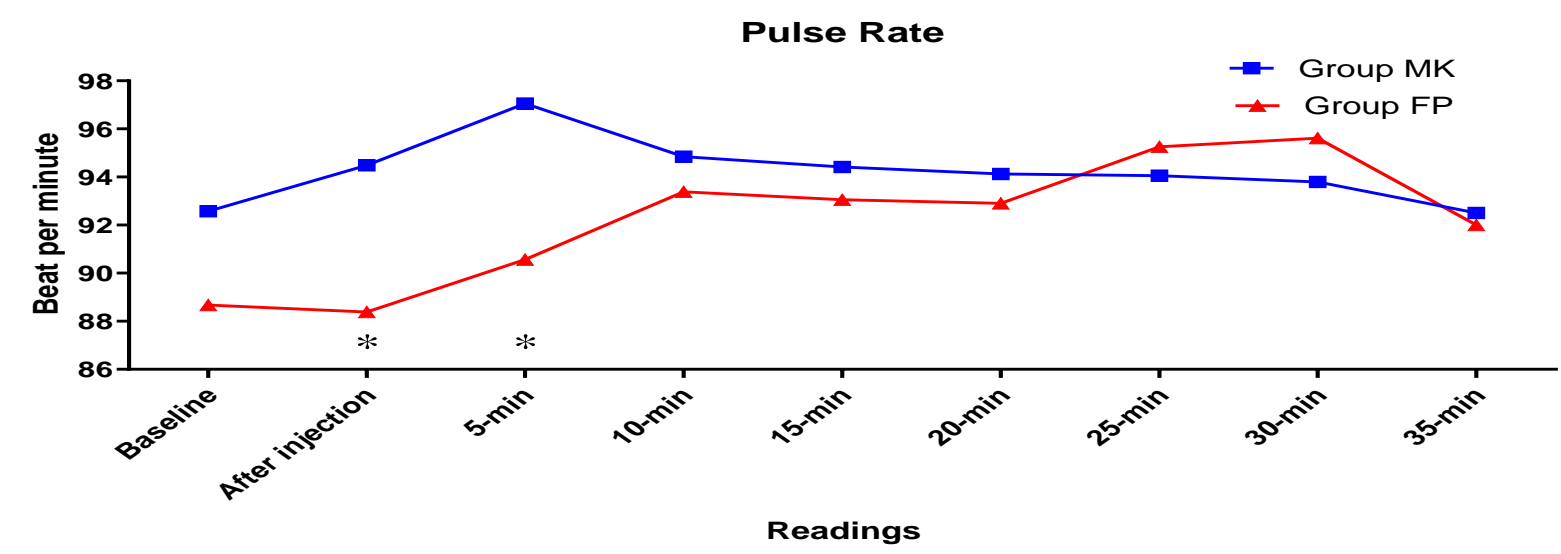

Figure (4): distribution of pulse rate at different intraoperative time intervals according to study groups (the asterisk indicates statistically significant difference, $p$-value $<0.05$ ) 
There were no statistically significant differences between study groups regarding SPO2, as shown in Figure (5).

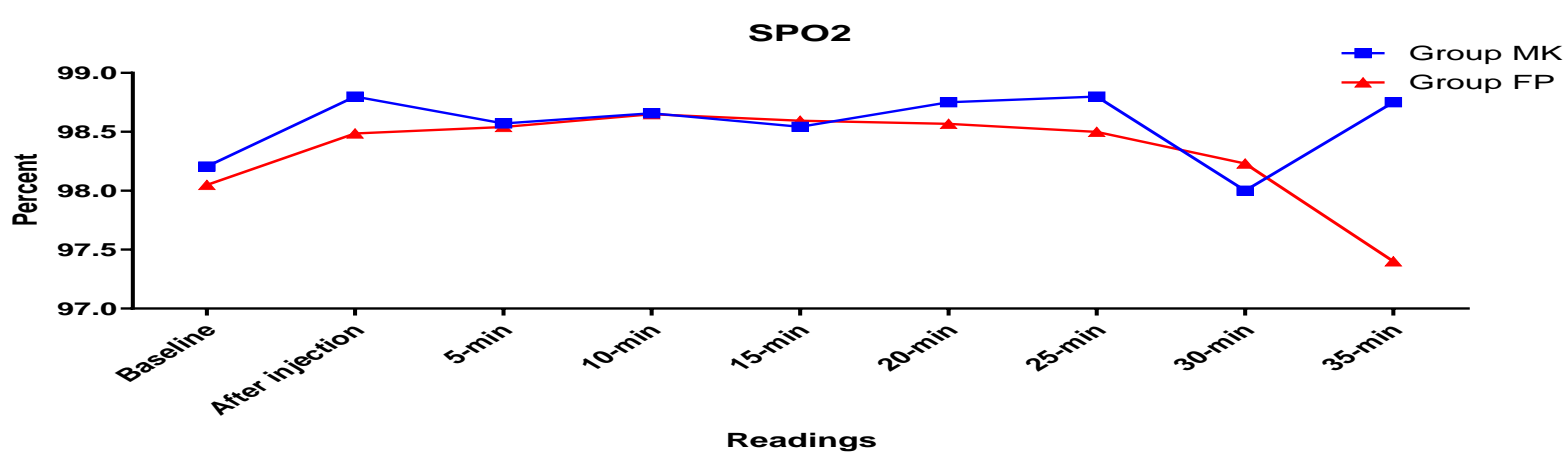

Figure (5): distribution of SPO2 at different intraoperative time intervals according to study groups (the asterisk indicates statistically significant difference, $p$-value $<0.05)$.

16The OAA/S score was significantly higher in group MK at most of examination times throughout anesthesia time, as shown in Figure (6).

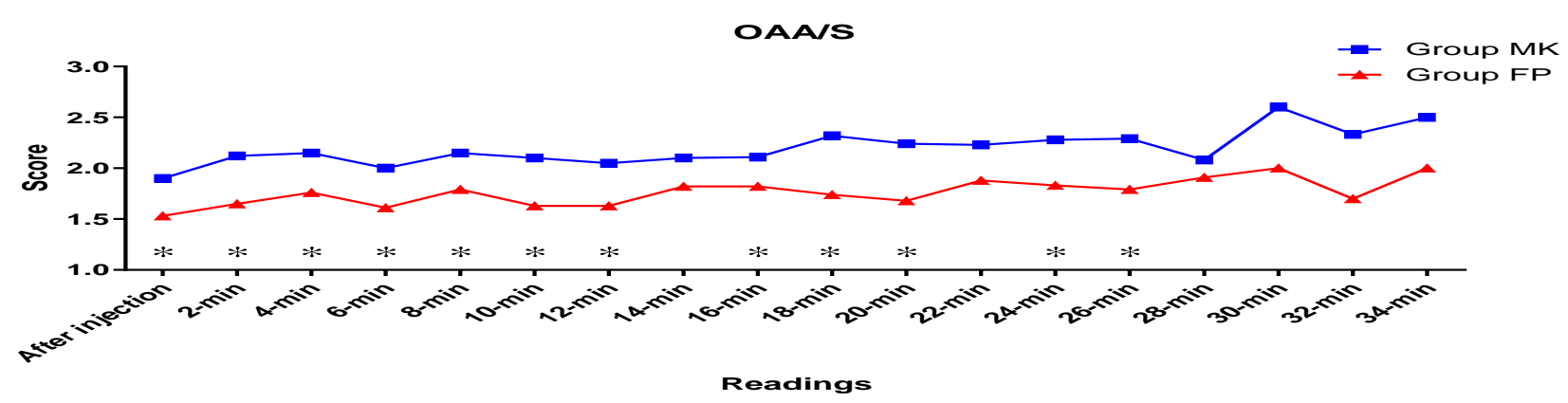

Figure (6): distribution of Observer's Assessment of Alertness/Sedation (OAA/S) score at different intraoperative time intervals according to study groups (the asterisk indicates statistically significant difference, $p$-value $<0.05$ )

\section{Discussion}

In current study, effective hypnoses were provided with (midazolam-ketamine) and (fentanyl-propofol) when performing an Endoscopic retrograde cholangiopancreatography (ERCP) in adults, the procedures performed with the fentanyl -propofol combination were more comfortable than that in the midazolam -ketamine group 
during the procedure. However, the time of recovery was significantly lower in group MK with compared to group FP, and discharge was significantly lower in group FP with, compared to in group MK. Concerning aside effect; MK showed slightly more side effects compared to group FP this led to more time for monitoring in recovery room and prolonged the discharge time. The ERCP is performed in operating theatre related to GIT unit. It suitable for general anesthesia. Moderate -deep sedation is generally used for this procedure. Therefore, we preferred sedation. In our study, deceased blood oxygen was occurred in one case MK, desaturation of oxygen was restored.

However, the finding of hypoxia was not statistically significant, and it can manage it by after providing additional oxygen. The emerging minor complication rate was low and the procedures were completed uneventful

In our study, the rate of complications through the end of procedure was markedly higher in the midazolam- ketamine group. Agitation, nausea and vomiting, headache and cough are significant side effects observed during recovery in ketamine-based sedation. Despite, it is known that opioids can induce postoperative nausea and vomiting, we did not observe this side effect in our study. None of the patients experienced serious problems in our study, similar to other studies, which reported only minor complications in the perioperative period ${ }^{(11,12,13) .}$ The prevalence of complications in both groups was the same whilst the kinds of complications were not the same in each group. Propofol has antiemetic effects so it would be expected that less nausea and vomiting occur in the FP group compared with opioids as shown in some other studies. Propofol is largely used for induce sedation in many ambulatory procedures. When propofol is used as a single agent for short term procedures, unwanted experiences and adverse effects may develop ${ }^{(12,13,14) .}$ Combining propofol with other medications may result in decreased dose of both drugs and their side effects. Concerning the analgesic effects, FP provides better sedation than $\mathrm{MK}^{(15)}$.

The OAA/S score was significantly higher in group MK at most of examination times throughout anesthesia time. so, Ketamine is regarded as a safe and effective sedative drug which had short time of effect, rapid onset, and has protection effect for $\mathbf{2 5}$ laryngeal reflexes (16,17). Despite these advantages, emergence phenomena (delusions, delirium, agitation, and discomfort dreams) is a problem when ketamine administered alone, given in large doses or rapidly administrations ${ }^{(18)}$. also the reports from some study suggest that concurrent administration of a benzodiazepine with ketamine 
decreases emergence reactions ${ }^{(19,20) .}$, while Sherwin reported that midazolam does not reduce recovery agitation and concluded that concurrent administration is unnecessary ${ }^{(21)}$. This difference can be due to slow IV injection of ketamine which can cause central apnea with rapid bolus ${ }^{(17)}$, According hemodynamic stability, the research showed that MK The heart rate were higher from the start of operation ketamine-induced hypertension and tachycardia. The systolic blood pressure did not show statistically significant differences through the anesthesia timeline, The diastolic BP started to be significantly lower in group FP compared to group MK. The MAP showed generally lower values through, the anesthesia, being significant at 10 and 15 minutes, and comparable stability between the groups. Several studies show effective of two-drug mixture of KM and FP are comfortable in middle of painful procedure include change of burning dress, primary orthopedic surgery, flexible fiberoptic. bronchoscope, alimentary system endoscope, cardinal catheterization, bone marrow aspiration, and lumbar puncture.as well as Mixing these two drugs leads to each one of their side effects alone and more rapid recovery. Fentanyl can be use in mixture with propofol for pain curing. Also, in mixing together, it has faster discharge period and less side adverse, in this study take more time for recovery. In contrast to the findings, some previous studies show that mixture of MK had a long recovery time and some others show that FP recovery period were longer than MK. This could be explained by the combinations and dosage.; Simsek et al. combined ketamine with midazolam. which has long recovery ${ }^{(22) .}$

Conclusion: current study concluded that FP, more suitable for sedation and less complications rather than MK. Although no significant complications were seen.

\section{References:}

(1) Adler DG, Baron TH, Davila RE, Egan J, Hirota WK, Leighton JA, Qureshi W, Rajan E, Zuckerman MJ, Fanelli R, Wheeler-Harbaugh J, Faigel DO; Standards of Practice Committee of American Society for Gastrointestinal Endoscopy. ASGE guideline: the role of ERCP in 
diseases of the biliary tract and the pancreas. Gastrointestinal Endosc. 2005.

(2) BRIEF ARTICLE Deep sedation during gastrointestinal endoscopy: Propofol- fentanyl and midazolam-fentanyl regimens WORLD JOURNAL OF GASTROENTEROLOGY World J Gastroenterol 2013 ,14; 19(22): 3439-3446

(3) ERCP Second Edition Todd H. Baron, MD, FASGE, Richard A. Kozarek, MD, FASGE,David L. Carr-Locke, MD, FRCP, FACG, FASGE 2013

(4) ERCP The Fundamentals Peter B. Cotton md fRCs fRCP ,Peter B. Cotton md fRCs fRCP, second edition 2015

(5) Kido K, Aoi A, Konno T, Yasuda M, Sato M, Shimoda H, et al. Balance Anaesthesia Using Sevoflurane and Fentanyl Based on Site Concentration Model Compared to Sevoflurane/N2O Anaesthesia for Oral Surgery. Anesth Prog. 2008;36:162-6.

(6) Iwakiri H, Nagata O, Matsukawa T, Ozaki M, Sessler DI. Effective concentration of propofol for recovery of consciousness is virtually independent of fentanyl Effectiveconcentration. Anesth Analg. 2003;96:1651-5.

(7) Hatamabadi HR, Arhami Dolatabadi A, Derakhshanfar H, Younesian S, Ghaffari Shad E. Propofol Versus Midazolam for procedural sedation of anterior shoulder Dislocation in Emergency Department: A Randomized Clinical Trial. Trauma Mon. 2015;20(2):13530.

(8) Ayatollahi V, Vafaiyan M, Hatami M, Behdad S. Two Different Concen- trations of Ketofol for Procedural Sedation and Analgesia in 
Closed Reduction of Nasal. Fracture. J Craniofac Surg. 2016;27(4):9961000.

(9) Vallejo MC, Romeo RC, Davis DJ, Ramanathan S. PropofolKetamine versus propofol-fentanyl for outpatient laparoscopy: Comparison of postoperative nausea, emesis, analgesia, and recovery. J Clin Anesth. 2002; 14:426-31.

(10) Watcha MF, Simeon RM, White PF, Stevens JL. Effect of propofol on the incidence of postoperative vomiting after strabismus surgery in pediatric outpatients. Anesthesiology. 1991;75:204-9.

(11) Weir PM, Munro HM, Reynolds PI, Lewis IH, Wilton NC. Propofol infusion and the incidence of emesis in pediatric outpatient strabismus surgery. Anesth Analg. 1993;76:760-4.

(12) Greves J, Glass PS, David A, Lubars KY, Matthew D, McEvoy, et al. Intravenous Anesthetic. In: Miller RD, editor. Anaesthesia. 7th ed. Philadelphia: Churchill Livingstone; 2010. pp. 746-7.

(13) Oncul S, Gaygusuz EA, Yilmaz M, Terzi H, Balci C. Comparison of ketamine-propofol and remifentanil in terms of hemody namic variables and patient satisfaction during monitored anaesthesia care. Anaesthesiol Intensive Ther. 2016;48(2):116-21.

(14) Nesher N, Serovian I, Marouani N, Chazan S, Weinbroum AA. Ketamine spares morphine consumption after transthoracic lung and heart 
surgery without adverse hemodynamic effects. Pharmacol Res. 2008;58:38-44.

(15) Miner JR, Biros M, Krieg S, Johnson C, Heegaard W, Plummer D, et al.,Randomized clinical trial of propofol versus methohexital for procedural sedation during fracture and dislocation reduction in the emergency department. Acad Emerg Med 2003;10:931-7.

(16). Marx CM, Stein J, Tyler MK, Nieder ML, Shurin SB, Blumer JL, et al. Ketamine-midazolam versus meperidine-midazolam for painful procedures in pediatric oncology patients. J Clin Oncol 1997;15:94-102.

(17) Gelen SA, Sarper N, Demirsoy U, Zengin E, Çakmak E. The efficacy and safety of procedural sedoanalgesia with midazolam and ketamine in pediatric hematology. Turk J Haematol 2015;32:351-4.

(18). Akhondzadeh R, Ghomeishi A, Nesioonpour S, Nourizade S. A com- parison between the effects of propofol-fentanyl with propofolketamine for sedation in patients undergoing endoscopic retrograde cholangiopancreatography outside the operating room. Biomed J. 2016;39(2):145-9.

(19) Kennedy RM, Porter FL, Miller JP, Jaffe DM. Comparison of fentanyl/midazolam with ketamine/midazolam for pediatric orthopedic emergencies. Pediatrics. 1998;102:956-63.

(20). Green SM, Johnson NE. Ketamine sedation for pediatric procedures: Part 2, review and implications. Ann Emerg Med. 1990;19:1033- 46. 
(21) Sherwin TS, Green SM, Khan A, Chapman DS, Dannenberg B. Does adjunctive midazolam reduce recovery agitation after ketamine sedation for pediatric procedures? A randomized, double-blind, placebo-controlled trial. Ann Emerg Med. 2000;35:229-38.

(22). Tülay D., Hilal S., Mehtap T., Şaziye Ş., and Aydın Y. A comparison of ketamine-midazolam and ketamine-propofol combinations used for sedation in the endobronchial ultrasound-guided transbronchial needle aspiration: a prospective, single-blind, randomized study. J Thorac Dis. 2014 Jun; 6(6): 742-751. 